\title{
Initiation of Isoniazid Prophylactic Therapy among Newly Diagnosed HIV-Positive Persons in Three High HIV-Burden Districts of South Africa
}

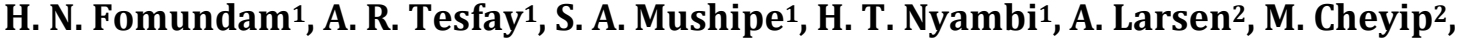 \\ G. Aynalem² ${ }^{2}$ A. K. Wutoh 1 \\ ${ }^{1}$ Howard University Global Initiative, Johannesburg, South Africa \\ ${ }^{2}$ US Centers for Disease Control and Prevention, Johannesburg, South Africa \\ Email: abrahamrezene@yahoo.com
}

How to cite this paper: Fomundam, H.N., Tesfay, A.R., Mushipe, S.A., Nyambi, H.T., Larsen, A., Cheyip, M., Aynalem, G. and Wutoh, A.K. (2020) Initiation of Isoniazid Prophylactic Therapy among Newly Diagnosed HIV-Positive Persons in Three High HIV-Burden Districts of South Africa. World Journal of AIDS, 10, 107-118. https://doi.org/10.4236/wja.2020.102009

Received: August 30, 2019

Accepted: May 12, 2020

Published: May 15, 2020

Copyright $\odot 2020$ by author(s) and Scientific Research Publishing Inc. This work is licensed under the Creative Commons Attribution International License (CC BY 4.0).

http://creativecommons.org/licenses/by/4.0/

\begin{abstract}
Background: South Africa is experiencing the worst HIV-driven tuberculosis (TB) epidemic in the world. More than 300,000 new cases of active TB are reported in the country each year with $60 \%$ co-infected with HIV. Isoniazid preventive therapy (IPT) is a key public health intervention for the prevention of TB among people living with HIV (PLHIV) and is recommended as part of a comprehensive HIV and AIDS care strategy. However, program data suggests that coverage of IPT service to be very low. This study aims to assess IPT initiation rate among newly diagnosed HIV-positive persons in three high HIV-burden districts of South Africa. Methods: A cross-sectional study was conducted using routine data generated from pre-ART and ART programs in 35 purposively selected primary health care (PHC) clinics in South Africa. The facilities were selected from three high HIV-burden districts with a mix of urban and rural settings. TB screening and IPT initiation status was assessed within a window period of one-year post HIV diagnosis. Initiation rate of IPT services among newly diagnosed HIV-positive persons was assessed. The chi-squared test was used to determine whether there was a significant difference in the proportion of newly diagnosed HIV-positive persons who were initiated on IPT by sex, age group, pregnancy status, health facility, district and location of facility. Results: We identified 12,413 newly diagnosed HIV patients aged 12-years-old and above between June 1, 2014 and March 31, 2015. TB screening was not conducted among 33\% of newly diagnosed HIV-positive persons to rule out or confirm the presence of active TB. IPT was initiated in $42.2 \%$ of known IPT-eligible HIV-positive persons. Initi-
\end{abstract}


ation of IPT services was lower in younger patients aged 12 to 20 -years-old compared to older patients. The proportion of pregnant women who were initiated on IPT was higher compared to the proportion in non-pregnant women $(51.0 \%$ and $40.1 \%$ respectively; $\mathrm{P}<0.05)$. Health care clinics located in uThukela health district registered the highest initiation rates of IPT care (48.1\%; CI: $46.2 \%, 50.1 \%$ ) compared to clinics located in Gert Sibande (40.4\%; CI: 38.7\%, 42.2\%) health district and Johannesburg Health District (38.5\%; CI: $36.4 \%, 40.7 \%)$. Conclusion: This analysis shows that initiation rate of IPT services among newly diagnosed HIV positive persons was low in the 35 participating facilities during the period under investigation. There was variability in IPT initiation rates across the facilities included in this study and among different sub-groups of the study sample. This study has identified specific population groups and geographic settings that should be targeted by programs to improve IPT services. There is a need to identify factors that contributed to the low initiation rate of IPT services among young HIV positive persons, women with unrecorded pregnancy status and in facilities located in inner city of Johannesburg. Customized interventions tailored to the specific needs of facilities and population groups should be instituted to strengthen uptake of IPT services.

\section{Keywords}

Isoniazid Prophylaxis Therapy, TB, HIV

\section{Background}

An estimated 6.8 million people are living with HIV (PLHIV) [1] [2] and HIV accounts for about two-thirds of hospital admissions in South Africa [3]. South Africa is also experiencing the worst HIV-driven tuberculosis (TB) epidemic in the world as about $1 \%$ of the general populations develop active TB each year. It ranks fourth in absolute number of notified TB cases, after India, China and Indonesia [4]. Relative to the size of the population, South Africa has the highest prevalence of TB in the world [4]. More than 300,000 new cases of TB are reported in the country each year with the HIV prevalence among TB patients at $60 \%$ [5]. Identifying strategies to improve TB prevention, particularly among $\mathrm{HIV}$-infected individuals is key to reducing HIV-related hospitalizations and deaths [6].

IPT is a key public health intervention for the prevention of TB among PLHIV and has been recommended as part of a comprehensive HIV and AIDS care strategy [7]. Several studies have shown that IPT reduces the risk of active TB by $33 \%$ overall and by $64 \%$ when targeted to HIV infected individuals who had a positive tuberculin skin test (TST) [8]. The combined effect of ART and IPT in preventing TB among PLHIV is significantly higher compared to ART alone [9]. There is evidence that co-administration of IPT and ART can reduce TB incidence in South Africa by more than $80 \%$ [9]. 
In South Africa, IPT is recommended as TB prophylaxis for all HIV-positive adults and adolescents in which active TB can be reasonable ruled out [6]. A six-question screening checklist has been developed to screen for active TB and determine eligibility for IPT service in HIV-positive persons at diagnosis and at every subsequent clinic visit [6]

IPT programs are poorly implemented globally partly due to lack of policy clarity experienced by healthcare providers [10]. The development and implementation of a national IPT policy remains suboptimal in most countries demonstrating high HIV/TB coinfection [10]. Limited availability of isoniazid and inadequate systems to manage drug supply impedes implementation of IPT services [10]). Insufficient health-care worker awareness due to lack of training and supervision, perceived low priority of IPT because of the absence of a reporting requirement, and fear that isoniazid prescription would identify patients as infected with HIV have been reported as the obstacles which impede the implementation of IPT [11]. Program data indicate there is poor uptake of IPT services among PLHIV. However, the extent of the gap in IPT update is not well documented. This study aims to assess the initiation rate of IPT services among newly diagnosed HIV-positive persons in selected primary health care facilities of South Africa and identify factors associated with low IPT initiation rate. In this study, initiation of IPT services is defined as receiving the first doses of isoniazid tables.

\section{Methods}

Study Design and setting: A cross-sectional study was conducted using data generated from pre-ART, ART and TB programs in 35 purposively selected primary health care (PHC) clinics. The clinics were selected from three high HIV-burden districts of South Africa (Gert Sibande, uThukela and City of Johannesburg). PHCs that could generate quality clinical data for newly diagnosed HIV-positive persons were identified in each district. PHC clinics were selected based on prevalence of HIV, HIV positivity rate and quality of clinical data available in the clinics.

Facilities were classified into rural, urban township, urban suburb or urban inner city based on the location of the facility. Facilities located in rural settlement away from cities and towns where agriculture is the mainstay of the economic activities were classified as "rural" facilities. Facilities located in central business district of an urban setting were classified as "urban inner city". Statistics South Africa classification of household income was used to further classify urban facilities into "Urban township" and "Urban suburb" [12]. Facilities located in a formal settlement of middle-income communities were classified as "urban townships". Facilities located in a suburb of upper income communities were classified as "urban suburb”.

Study Sample: All newly diagnosed HIV-positive individuals aged 12 years and above who received their first HIV-positive test results between June 1, 2014 
and March 31, 2015 in public facilities selected for the study and had medical records available for review were included in this study. All newly diagnosed HIV-positive persons were considered potentially eligible for IPT. However, only individuals for whom the presence of active TB was ruled out through TB screening and diagnostic services were included in this analysis.

Data Sources: Data on TB screening and IPT initiation was collected from various clinical registers and records through clinical record review using a standardized case record form. IPT initiation status was assessed within a period of one-year after HIV diagnosis through clinical record review between June 1, 2014 and December 2015. Data was collected by trained and independent data abstractors dedicated for this purpose.

Data Quality Management: Several measures were taken to ensure the quality of data used in this study and minimize information bias. Data quality assessments were conducted using a structured tool and clinics that scored below $90 \%$ on completeness and accuracy of clinical data were dropped from the study. Completeness was assessed based on availability of records on a set of data elements. Accuracy was assessed by contrasting clinical data elements across different registers. IPT initiation was assessed within a window period of one-year after establishing a new HIV diagnosis. Completed Case Record Forms (CRFs) were captured using Epi Info version 7.1.5 template by two independent data capturers to minimize data entry error. Computerized data quality verification was performed by contrasting the two independent data sets using STATA version 14.1 (College Station) software.

Statistical Analysis: Newly diagnosed HIV-positive persons were classified as "initiated on IPT regimen" if they completed IPT eligibility assessment based on the national IPT guidelines [10] and IPT was provided to those who were eligible. Proportion of newly diagnosed HIV-positive persons who were initiated on IPT was computed using STATA 14. Difference in the proportion of HIV-positive persons who were initiated on IPT was examined by sex, age group, pregnancy status, health facility, district and facility location using chi square test.

Ethical Considerations: The protocol for the pre-ART surveillance project was reviewed according to the Centers for Disease Control and Prevention (CDC) human research protection procedures and was determined to be a research, but $\mathrm{CDC}$ was not engaged. It was approved by the Research Ethics Committee at the School of Health Sciences and Public Health at University of Pretoria (protocol number 169/2013). Howard University also obtained relevant permissions from the National and the respective Provincial Departments of Health of South Africa.

\section{Results}

We identified 12,413 newly diagnosed HIV-positive persons in 35 PHC facilities between June 1, 2014 and March 31, 2015. A total of 16 PHC facilities were dropped due to poor data quality. Around 70\% of newly diagnosed persons were 
female. Most (72\%) of the study participants were aged between 21 and 40-years. Table 1 below illustrates demographic composition of the study sample in detail. About $94 \%$ of newly diagnosed HIV-positive persons were screened for active TB using a six-question TB screening checklist.

Figure 1 illustrates the cascade of newly diagnosed HIV-positive persons with respect to TB screening and IPT service provision. TB screening service was documented for nearly two-third (64\%) of all newly diagnosed HIV-positive persons. The presence of active TB was ruled out in $95 \%$ of those who had documented TB screening outcome, establishing eligibility for IPT based on the national guideline [6]. Less than half (42\%) of the HIV-positive persons who were known to be eligible for IPT were initiated on IPT care within 12 months of diagnosis. About $5 \%$ of the HIV-positive persons with documented TB screening outcome were either suspected or confirmed to have active TB and were not considered for IPT initiation.

There was significant variability in the initiation of IPT care in IPT eligible HIV-positive persons across age categories. There was no statistically significant difference in the proportion of male and female patients who were initiated on

Table 1. Newly diagnosed HIV-positive persons who were initiated on IPT care.

\begin{tabular}{|c|c|c|c|c|c|c|}
\hline & \multirow{2}{*}{ Variables } & \multirow{2}{*}{$\begin{array}{c}\text { Total Newly } \\
\text { Diagnosed } \\
\text { HIV-Positive } \\
\text { Persons Eligible } \\
\text { for IPT }(\mathrm{N}=7773)\end{array}$} & \multicolumn{3}{|c|}{$\begin{array}{l}\text { IPT Initiation Status } \\
\qquad(\mathrm{N}=7773)\end{array}$} & \multirow{2}{*}{ C.I. } \\
\hline & & & No & Yes & $\begin{array}{c}\% \\
\text { Initiated }\end{array}$ & \\
\hline \multirow[t]{3}{*}{ Sex } & Female & 5483 & 3128 & 2355 & $43.00 \%$ & $(41.6 \%, 44.3 \%)$ \\
\hline & Male & 2290 & 1343 & 947 & $41.40 \%$ & $(39.3 \%, 43.4 \%)$ \\
\hline & Total & 7773 & 4471 & 3302 & $42.50 \%$ & $(41.4 \%, 43.6 \%)$ \\
\hline \multirow{5}{*}{$\begin{array}{c}\text { Age } \\
\text { Category }\end{array}$} & $12-20$ & 705 & 442 & 263 & $37.30 \%$ & $(33.7 \%, 41.0 \%$ \\
\hline & $21-30$ & 3365 & 1923 & 1442 & $42.90 \%$ & $(41.2 \%, 44.5 \%)$ \\
\hline & $31-40$ & 2339 & 1314 & 1025 & $43.80 \%$ & $(41.8 \%, 45.9 \%)$ \\
\hline & $41-50$ & 965 & 561 & 404 & $41.90 \%$ & $(38.7 \%, 45.1 \%)$ \\
\hline & $>50$ & 399 & 231 & 168 & $42.10 \%$ & $(37.2 \%, 47.1 \%)$ \\
\hline \multirow{3}{*}{$\begin{array}{c}\text { Pregnancy } \\
\text { Status } \\
\text { (Female) }\end{array}$} & Pregnant & 1752 & 859 & 893 & $51.00 \%$ & $(48.6 \%, 53.3 \%)$ \\
\hline & Non-pregnant & 3421 & 2050 & 1371 & $40.10 \%$ & $(38.4 \%, 41.7 \%)$ \\
\hline & Not recorded & 310 & 219 & 91 & $29.40 \%$ & $(24.3 \%, 34.8 \%)$ \\
\hline \multirow[t]{3}{*}{ District } & uThukela & 2581 & 1339 & 1242 & $48.10 \%$ & $(46.2 \%, 50.1 \%)$ \\
\hline & Gert Sibande & 3141 & 1871 & 1270 & $40.40 \%$ & $(38.7 \%, 42.2 \%)$ \\
\hline & Johannesburg & 2051 & 1261 & 790 & $38.50 \%$ & $(36.4 \%, 40.7 \%)$ \\
\hline \multirow{4}{*}{$\begin{array}{l}\text { Clinic } \\
\text { Setting }\end{array}$} & Urban Inner City & 303 & 288 & 15 & $5.00 \%$ & $(2.8 \%, 8.0 \%)$ \\
\hline & Urban Township & 4932 & 3103 & 1829 & $37.10 \%$ & $(35.7 \%, 38.4 \%)$ \\
\hline & Rural & 1307 & 693 & 614 & $47.00 \%$ & $(44.2 \%, 49.7 \%)$ \\
\hline & Urban Suburb & 1231 & 387 & 844 & $68.60 \%$ & $(65.9 \%, 71.1 \%)$ \\
\hline
\end{tabular}




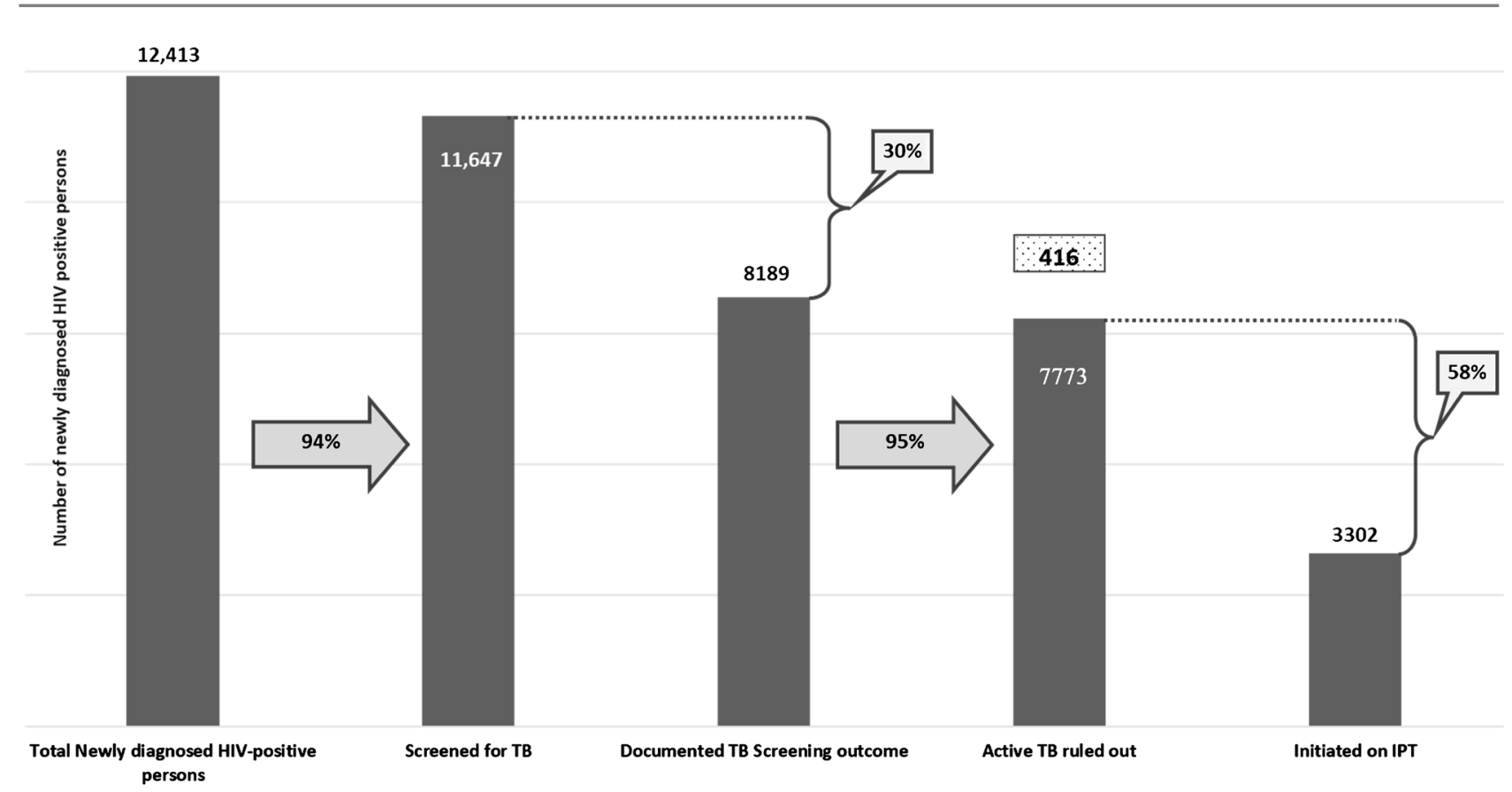

Figure 1. Cascade of TB screening and IPT service provision for newly diagnosed HIV-positive persons $(\mathrm{N}=12,413)$.

IPT ( $41.4 \%$ and $43.0 \%$ respectively). The proportion of pregnant women who were initiated on IPT care was significantly higher than the proportion in non-pregnant women $(51.0 \% \%$ and $40.1 \%$, respectively).

The clinics selected from Johannesburg Health District had the lowest initiation rate of IPT service (38.5\%), when compared to clinics selected from Gert Sibande and uThukela health districts $(40.4 \%$ and $48.1 \%)$, though the difference between Johannesburg health district and Gert Sibande health district is not statistically significant. Clinics located in urban suburbs had the highest rates of IPT initiation at $68.4 \%$. Conversely, clinics located in inner cities, which were located only in Johannesburg Health District, had the lowest IPT initiation rates (5\%), though the sample size was small and the measurement may not be precise.

Further sub-analysis showed significant variability in the proportion of newly diagnosed HIV-positive persons who were initiated on IPT service across the 35 selected clinics. The lowest IPT initiation rate among IPT eligible newly diagnosed HIV positive persons was observed in a clinic selected from Johannesburg Health District Region E in which only 4\% of 303 IPT eligible newly diagnosed HIV-positive persons were initiated on IPT. The highest IPT initiation rate was observed in a clinic selected from Gert Sibande Health District and had an IPT initiation rate of $89 \%$ among 101 IPT eligible newly diagnosed HIV-positive persons. None of the selected clinics reached $90 \%$ of IPT initiation rate and more than half of the clinics did not reach a 50\% IPT initiation rate among IPT eligible newly diagnosed HIV-positive persons.

\section{Limitations}

This study involved analysis of data that include medical records and registers of 
newly diagnosed HIV positive persons in selected public facilities during the study timeframe. Participating facilities were selected based on purposive sampling technique. This analysis involves large sample size drawn from diverse facilities from urban, peri-urban and rural settings; however, the sample represents only 3 of 52 districts in South Africa and does not include private facilities. As such, the study population may not be representative of the total population of newly diagnosed HIV-positive persons in South Africa. This analysis may provide clues on the level of IPT initiation among newly diagnosed HIV-positive persons. However, the findings of this study may not be directly extrapolated to newly diagnosed persons not included in the study.

TB screening services could not be determined for about one-third of newly diagnosed HIV-positive persons due to missing clinical data. Exclusion of these individuals from the analysis could have influenced the observed outcome in either direction depending on the distribution of missing data in the study population. This analysis is based on secondary data generated during routine clinical management of newly diagnosed HIV-positive persons at participating facilities. The investigators could not independently ascertain the provision or lack of services provided to HIV-positive persons. An assumption was made that clinical records reflected service delivery. Clinical records contain minimal data on characteristics of individual patients and this limited the researchers in determining the other factors associated with IPT initiation.

A study design that involves probability sampling technique when selecting newly diagnosed HIV-positive persons and active collection of primary data instead of passively relying on clinical records could have improved the validity of this study and address the issue of missing data.

\section{Discussion}

South African guideline for tuberculosis preventive therapy recommends that PLHIV should be screened for TB during each clinical visit [10]. This study shows that $94 \%$ of newly diagnosed HIV-positive persons were screened for the presence of active TB while completing HIV testing service (HTS). However, TB screening outcomes were not documented in the clinical record for about $30 \%$ of newly diagnosed HIV-positive persons. Most newly diagnosed HIV-positive persons with a documented TB screening outcome, screened negative (95\%), ruling out active TB and establishing eligibility for an IPT service. Only $2.9 \%$ of the cohort of newly diagnosed HIV-positive persons presented with active TB during HIV diagnosis.

IPT is an effective public health intervention for the prevention of active TB infections among PLHIV [6]. Despite being life-saving for PLHIV and that the majority of newly diagnosed PLHIV were eligible, this study shows that the initiation rate of IPT service among newly diagnosed HIV-positive persons is sub-optimal. PLHIV who do not receive sustained ART services and complete the recommended IPT regimen are at much greater risk of developing active TB 
during the advanced stages of HIV infection [9].

Program data from PEPFAR-supported clinics indicated only $1 \%$ of potentially IPT eligible HIV patients were initiated on IPT in 2010 [13]. An updated guideline for IPT service was released in 2010, which was meant to be implemented in selected sites beginning April 2010 and expand its implementation in subsequent years [14]. The guideline recommended a 6-month IPT for all HIV-infected persons, regardless of TST status [14]. The proportion of IPT eligible HIV patients who were initiated on IPT rose to $10.5 \%$ within a year in selected facilities [13].

This study shows that IPT was initiated in $42.5 \%$ of the IPT eligible HIVpositive persons overall and wide variability was observed across the facilities and age groups. A third of newly diagnosed HIV-positive persons did not enrol for HIV care in the health care facility where HIV diagnosis was confirmed and did not have an established IPT eligibility status. Several other studies have reported similar observations. Data from high-burden, resource-limited countries suggest that uptake and adherence to IPT is sub-optimal [15] as only $24 \%$ to $59 \%$ of HIV patients complete the recommended IPT regimen [16]. Review of IPT services in Uganda, South Africa, and Botswana showed sub-optimal completion rate of IPT regimen [17] and similar study in Kenya reported that uptake of IPT services was very low (33\% - 40\%) [17]. WHO estimates only 933,000 PLHIV received IPT service in 2014 [18].

The observed low initiation rate of IPT services can be explained by factors influencing the outcome at different levels of the health care system that include the policy environment, the health care provider and the patient [7] [19] [20] [21]. Engagement of newly diagnosed HIV-positive persons with the health care system is critical to improve initiation of IPT. This study shows one-third of newly diagnosed HIV-positive persons were not effectively linked to the continuum of HIV care after completing the HTS. Most of the attritions from HIV care occurred during the early period after HIV diagnosis before newly diagnosed HIV-positive persons could be enrolled in the ART or pre-ART programs [12]. Early loss to follow-up is a major challenge among newly diagnosed HIV-positive persons and occurs due to supply and/or demand side barriers to care [7] [17] [18]. TB screening outcome was not documented for most individuals who drop out of care in the early phase of the continuum of HIV care. Most HIV-positive persons dropped out of care after HIV diagnosis. These patients usually return to care only when they are very sick with advanced HIV infection and severely compromised immune system, elevating the risk of developing active TB [22] Perceived judgmental attitude of health care providers, fear and social stigma associated with testing HIV-positive and high travel costs reduce the motivation of newly diagnosed HIV-positive individuals to engage with the health care system [23]. Disclosure of HIV-positive status to immediate family members and a supportive clinical environment could encourage retention in HIV care and improve initiation of IPT services [23]. A trial conducted in Tanzania demonstrat- 
ed that an IPT initiation rate of up to $90 \%$ and IPT completion rate of $87 \%$ could be achieved if HIV-infected individuals receive counselling services, monthly follow-up and travel reimbursement [24] to encourage linkage and retention in care. Patients who are aware of their elevated risk of developing active TB and who understand the benefits of IPT are more likely to initiate and adhere to the IPT regimen [14].

Significant variability in the initiation rate of IPT services was observed among newly diagnosed HIV-positive persons across the 35 clinics. A few clinics performed very well and registered an IPT initiation rate of $80 \%$ and above among HIV-positive clients who were screened and known to be eligible for IPT service, while others performed poorly registering an IPT initiation rate of less than $10 \%$. Differences in the initiation rates of IPT services across clinics may demonstrate variation in human and material resources to effectively implement the guideline [13]. With the advent of HIV and TB epidemics, most primary health care facilities in South Africa are stretched beyond their capacity [25]. They lack adequately trained health care personnel and other resources to meet the needs of hundreds of patients who visit their health care facilities [25]. This has precipitated overcrowding and long waiting hours in public health care facilities which may further diminish staff moral and resulted in poor patient-provider interactions and inadequate counseling services [7]. Inadequate capacity at facility level to provide sufficient information on the risk of TB and the benefit of IPT services to HIV-patients can be a significant barrier to the uptake of IPT services [14].

Health care providers who are not familiar with the benefits and recommendations outlined in the guideline may not have the confidence and motivation necessary to implement IPT programs. Some health care providers may need more training on the benefits of IPT and procedures for administering IPT services to address their concerns about toxicity of isoniazid and maintaining adherence to the IPT regimen. A quality improvement intervention that involved brief training and the use of TB screening checklist resulted in significant improvement in TB screening rates and initiation of IPT in HIV-positive patients in Ethiopia [25]. Substantial integration of TB and HIV services also improves the performance of the IPT program [26] [27].

Initiation rate of IPT services was lower in clinics selected from Johannesburg Health District (38.5\%) compared to the levels observed in clinics selected from Gert Sibande and uThukela Health Districts ( $40.4 \%$ and $48.1 \%$ respectively), though the difference between Johannesburg health district and Gert Sibande health district was not statistically significant. Clinics located in inner cities (4.7\%) and urban townships (36.8\%) registered much lower initiation rate of IPT services compared to clinics located in rural areas (46.7\%) and middle-class suburbs (68.4\%). Most of the study health care facilities in Johannesburg Health District are located in townships and the inner city. Another possible factor could be that a significant fraction of the population served by selected clinics in 
Johannesburg area are mobile population who migrated from their habitual rural areas in search of temporary work and other opportunities. Most of the clinics do not cater for the special needs of mobile populations including extending operational hours and providing services over the weekends and holidays [25]. This may discourage mobile people from engaging with the health care system and thereby inhibiting initiation of IPT care.

\section{Conclusion and Recommendation}

This analysis shows initiation of IPT services in the selected facilities was very low during the period under investigation. The lowest IPT initiation rates were observed in HIV-positive persons aged between 12 to 20 years, females for whom pregnancy status was not recorded and in facilities located in the inner city of Johannesburg. Research is needed to identify factors that contributed to the low initiation rate of IPT in these sub-populations. Programs should consider interventions that are targeted to the specific needs of these population groups and facilities.

\section{Acknowledgements}

This research work was made possible through the support provided by the President's Emergency Plan for AIDS Relief through Centers for Disease Control and Prevention under Cooperative Agreement (Grant number 5U2GGH000391). The funder provided technical oversight during the development of the study design, data analysis and during the preparation of this manuscript.

\section{Disclaimer}

The findings and conclusions expressed in this paper are those of the authors and do not necessarily represent the official position of the funding agencies.

\section{Conflicts of Interest}

The authors declare no conflicts of interest regarding the publication of this paper.

\section{References}

[1] United Nations (2014) United Nations Joint Programme on HIV/AIDS (UNAIDS) TGR.

[2] Shisana, O., Rehle, T., Simbayi, L., Zuma, K., Jooste, S. and Zungu, N. (2012) South African National HIV Prevalence, Incidence and Behavioural Survey. Human Science Research Council (HSRC) Cape Town, HSRC Press, Pretoria.

[3] Meintjes, G., Kerkhoff, A.D., Burton, R., Schutz, C., Boulle, A., Van Wyk, G., et al. (2015) HIV-Related Medical Admissions to a South African District Hospital Remain Frequent Despite Effective Antiretroviral Therapy Scale-Up. Medicine, 94, e2269. https://doi.org/10.1097/MD.0000000000002269

[4] World Health Organisation (2015) Global Tuberculosis Report 2015. World Health Organisation, France. 
[5] World Health Organisation (2014) Global Tuberculosis Report 2014. World Health Organisation, France.

[6] National Department of Health (2014) National Consolidated Guidelines for the Prevention of Mother-to-Child Transmission of HIV (PMTCT) and the Management of HIV in Children Adolescents and Adults. National Department of Health, South Africa.

[7] Mindachew, M., Deriew, A., Memiah, P. and Biadgilign, S. (2014) Perceived Barriers to the Implementation of Isoniazid Preventive Therapy for People Living with HIV in Resource Constrained Settings. Pan African Medical Journal, 17, 2641. https://doi.org/10.11604/pamj.2014.17.26.2641

[8] Golub, J.E., Lerato, M., Nkeko, T. and Mosa, M. (2009) Isoniazid Preventive Therapy, HAART and Tuberculosis Risk in HIV-Infected Adults in South Africa: A Prospective Cohort. AIDS, 23, 631-636. https://doi.org/10.1097/QAD.0b013e328327964f

[9] Akolo, C., Bada, F. and Okpokoro, E. (2015) Debunking the Myths Perpetuating Low Implementation of Isoniazid Preventive Therapy amongst Human Immunodeficiency Virus-Infected Persons. World Journal of Virology, 4, 105 -112. https://doi.org/10.5501/wjv.v4.i2.105

[10] National Department of Health (2010) Guidelines for Tuberculosis Preventive Therapy among HIV Infected Individuals. National Department of Health, South Africa.

[11] Raizada, N., Chauhan, L.S., Babu, B.S., Thakur, R., Khera, A., Wares, F.D., et al. (2009) Linking HIV infected TB patients to Cotrimoxazole Prophylaxis and Antiretroviral Treatment in India. PLoS ONE, 4, e5999. https://doi.org/10.1371/journal.pone.0005999

[12] World Health Organisation (2015) Global Health Sector Response to HIV, 2000-2015 Focus on Innovations in Africa. World Health Organisation, France.

[13] Bristow, C.C., Larson, E., Vilakazi-Nhlapo, A.K., Wilson, M. and Klausner, J.D. (2012) Scale-up of Isoniazid Preventive Therapy in PEPFAR-Assisted Clinical Sites in South Africa. The International Journal of Tuberculosis and Lung Disease, 16, 1020-1022. https://doi.org/10.5588/ijtld.11.0744

[14] Churchyard, G.J., Mametja, L.D., Mvusi, L., Ndjeka, N., Hesseling, A.C., Reid, A., et al. (2014) Tuberculosis in South Africa; Success, Challenges and Recommendations. The South African Medical Journal, 104, 244-248. https://doi.org/10.7196/SAMJ.7689

[15] World Health Organisation (2004) Report of a "Lessons Learnt" Workshop on the Six ProTEST Pilot Projects in Malawi, South Africa, and Zambia. WHO, Geneva.

[16] Uyei, J., Coetzee, D., Macinko, J. and Guttmacher, S. (2011) Integrated Delivery of HIV and Tuberculosis Services in Sub-Saharan Africa: A Systematic Review. The Lancet Infectious Diseases, 11, 855-857. https://doi.org/10.1016/S1473-3099(11)70145-1

[17] World Health Organisation (2015) Global Tuberculosis Report 2015. Report No. WHO/HTM/TB/2015.22. World Health Organization, Geneva.

[18] Teklay, G., Tsigemariam, T., Befikadu, L., Tedla, K. and Klinkenberg, E. (2016) Barriers in the Implementation of Isoniazid Preventive Therapy for People Living with HIV in Northern Ethiopia: A Mixed Quantitative and Qualitative Study. BMC Public Health, 19, 840. https://doi.org/10.1186/s12889-016-3525-8

[19] Statistics South Africa (2017) Living Conditions of Households in South Africa. An Analysis of Household Expenditure and Income Data Using the LCS 2014/2015. Pretoria. 
[20] Bakari, M., Moshi, A., Aris, E.A., Chale, S., Josiah, R., Magao, P., et al. (2000) Isoniazid Prophylaxis for Tuberculosis Prevention among HIV Infected Police Officers in Dares Salaam. East African Medical Journal, 77, 494 -497. https://doi.org/10.4314/eamj.v77i9.46695

[21] Amin, S., Hassan, O., Fielding, K.L., Nahashon, M.T., Nabwera, H.M., Sanders, E.J., et al. (2012) Early Loss to Follow-up of Recently Diagnosed HIV-Infected Adults from Routine Pre-ART Care in a Rural District Hospital in Kenya: A Cohort Study. Tropical Medicine \& International Health, 17, 82 -93. https://doi.org/10.1111/j.1365-3156.2011.02889.x

[22] Larson, B.A., Brennan, A., McNamara, L., Long, L., Rosen, S., Sanne, I., et al. (2010) Early Loss to Follow up after Enrolment in Pre-ART Care at a Large Public Clinic in Johannesburg, South Africa. Tropical Medicine \& International Health, 15, 43-47. https://doi.org/10.1111/j.1365-3156.2010.02511.x

[23] Zaeh, S., Russell Kempker, R., Edward Stenehjem, E., Blumberg, H.M., Temesgen, O.O.I. and Tenna, A. (2013) Improving Tuberculosis Screening and Isoniazid Preventative Therapy in an HIV Clinic in Addis Ababa, Ethiopia. International Journal of Tuberculosis and Lung Disease, 17, 1396-1401. https://doi.org/10.5588/ijtld.13.0315

[24] Rowe, K.A., Makhubele, B., Hargreaves, J.R., Porter, J.D., Hausler, H.P. and Pronyk, P.M. (2005) Adherence to TB Preventive Therapy for HIV Positive Patients in Rural South Africa: Implications for Antiretroviral Delivery in Resource-Poor Settings. International Journal of Tuberculosis and Lung Disease, 9, 263-269.

[25] Fomundam, H.N., Wutoh, A., Tesfay, A., Boshielo, T.C., Maranga, A., Mushipe, S.A., et al. (2013) Surveillance of HIV-Positive Pre-ART Persons in South Africa: Gap Analysis Report. Howard University, Pretoria, South Africa.

[26] Lisa, V., Elizabeth, A., Odato, K., Blunt, H. and Steingart, K.R. (2014) Interventions to Improve Delivery of Isoniazid Preventive Therapy: An Overview of Systematic Reviews. BMC Infectious Diseases, 14, 281. https://doi.org/10.1186/1471-2334-14-281

[27] Chemhuru, M., Tshimanga, M. and Tafara, N. (2015) Evaluation of the Isoniazid Preventive Therapy (IPT) Program in Shurugwi District, Midlands Province, Zimbabwe, January 2013 to August 2014. BMC Research, 8, 476.

https://doi.org/10.1186/s13104-015-1451-y 